\title{
Rhein, an Active Metabolite of Diacerein, Suppresses the Interleukin- $1 \alpha$-Induced Proteoglycan Degradation in Cultured Rabbit Articular Chondrocytes
}

\author{
Tadafumi Tamura* and Kenji Ohmori \\ Pharmaceutical Research Institute, Kyowa Hakko Kogyo Co., Ltd., Shimotogari, Nagaizumi, Sunto, Shizuoka 411-8731, Japan
}

Received September 20, 2000 Accepted November 8, 2000

\begin{abstract}
Diacerein has proved to be effective in the treatment of osteoarthritis. We investigated the effects of rhein, an active metabolite of diacerein, on the degradation of recombinant human interleukin- $1 \alpha$ (rhIL-1 $\alpha$ )-induced proteoglycan and matrix metalloproteinases (MMPs) release from rabbit articular chondrocytes. Chondrocytes were treated for $24 \mathrm{~h}$ with rhein in the presence of rhIL-1 $\alpha$. Rhein suppressed the rhIL-1 $\alpha$-induced proteoglycan degradation. In addition, rhein decreased the rhIL- $1 \alpha$-induced proMMP-3 production and reduced the MMPs activity. These results suggested that diacerein might have a protective effect on proteoglycan degradation via the suppressive effect of rhein on the production of proMMPs.
\end{abstract}

Keywords: Diacerein, Rhein, Chondroprotection

Osteoarthritis $(\mathrm{OA})$ is characterized by the destruction of articular cartilage. The extracellular matrix of articular cartilage is composed mainly of collagen and proteoglycan aggregates. In the case of articular diseases such as OA, an alteration of the articular chondrocyte metabolism occurs, resulting in breakdown of the cartilage matrix. Currently, the primary approach in the clinical treatment of OA involves the use of nonsteroidal anti-inflammatory drugs (NSAIDs), analgesics and hyaluronan, which allow symptomatic relief, but provide no apparent disease-modifying effect. In some instances, NSAIDs may even be deleterious; they inhibited the synthesis of proteoglycan, which plays a crucial role in maintaining the function of the cartilage. Therefore, there is a critical need to develop alternative agents that prevent the destruction of cartilage and/or stimulate its proper repair.

Diacerein has proven to be effective and well tolerated in the long-term treatment of $\mathrm{OA}$, and it is currently under evaluation as a disease modifying $\mathrm{OA}$ drug in randomized placebo-controlled clinical trials of patients with OA of the hip and knee $(1,2)$. The efficacy of diacerein has been documented in animal models, including the accelerated canine model of OA (3). Diacerein has a protective effect on granuloma-induced cartilage breakdown by a reduction in the concentrations of proinflammatory cytokines (4). However,

*Corresponding author. FAX: +81-559-86-7430

E-mail: tadafumi.tamura@kyowa.co.jp the exact mechanisms of therapeutic action of diacerein on $\mathrm{OA}$ are not clear. To obtain further insight into the effects of diacerein, monolayer cultures of rabbit articular chondrocytes were used to investigate the effect of rhein, an active metabolite of diacerein (5), on recombinant human IL-1 $\alpha($ rhIL- $1 \alpha)$-induced proteoglycan degradation and the production of promatrix metalloproteinases (proMMPs).

Articular chondrocytes were prepared from the shoulder and knee joints of 3-week-old female Japanese White rabbits (Tokyo Experimental Animal, Tokyo). Chondrocytes isolated by treatment with collagenase were cultured in $100-\mathrm{mm}$ culture dishes at a density of $2.5 \times 10^{4} \mathrm{cells} / \mathrm{cm}^{2}$ in Dulbecco's modified Eagle's medium (DMEM; Life Technologies Inc., Rockville, MD, USA) with 10\% fetal bovine serum (BioWhittaker, Walkersville, MD, USA) and antibiotics $(100 \mathrm{U} / \mathrm{ml}$ penicillin, $100 \mu \mathrm{g} / \mathrm{ml}$ streptomycin and $250 \mathrm{ng} / \mathrm{ml}$ fungizone ${ }^{\mathbb{B}}$; Life Technologies Inc.) in $5 \%$ $\mathrm{CO}_{2}-95 \%$ air at $37^{\circ} \mathrm{C}$. Primary cultures maintained in a monolayer were used throughout the study. After the chondrocytes reached confluence, the medium was changed to DMEM with $0.2 \%$ lactalbumin hydrolysate (Sigma Chemical, St. Louis, MO, USA) and the antibiotics. Confluent chondrocytes were then incubated with rhein sodium salt (Kyowa Hakko, Tokyo) or naproxen (Sigma) in the presence of rhIL-1 $\alpha$ (Genzyme, Cambridge, MA, USA) for $24 \mathrm{~h}$. The concentration of rhIL-1 $\alpha$, at which significant sulfated glycosaminoglycan (S-GAG) release and proMMPs production were obtained, was determined in a pre- 
liminary experiment.Recombinant hIL-1 $\alpha(0.1-10 \mathrm{ng} / \mathrm{ml})$ was tested, and the concentration of $1 \mathrm{ng} / \mathrm{ml}$ was selected. Rhein sodium salt was dissolved in distilled water at $3 \times 10^{-2} \mathrm{M}$ just before each experiment, and naproxen was dissolved in dimethyl sulfoxide at $1 \times 10^{-1} \mathrm{M}$; they were added to the cell culture at the appropriate concentrations. The final concentrations of distilled water and dimethyl sulfoxide were $0.1 \%$ and $0.01 \%$, respectively. The concentrations of rhein were chosen to reflect levels that are below, at or above the established therapeutic anti-inflammatory serum values of rhein after dosing with diacerein described in clinical practice (6). The harvested culture media were stored at $-40^{\circ} \mathrm{C}$ until use. Rhein and naproxen had no effect on chondrocyte viability in this study.

The destruction of articular cartilage results from a failure of chondrocytes to maintain a homeostatic balance between matrix synthesis and degradation. Proinflammatory cytokines such as IL-1, which are produced by monocytes /macrophages, synovial cells or chondrocytes, are thought to play major roles in joint diseases such as OA or rheumatoid arthritis (RA) $(7,8)$. IL-1 has been shown to inhibit extracellular matrix synthesis and stimulate proteoglycan degradation (7). In this communication, IL-1 $\alpha$-induced proteoglycan degradation was assessed by measuring the amount of S-GAG released from chondrocytes into the culture medium. S-GAG was determined using 1,9-dimethylmethylene blue (Aldrich, Milwaukee, WI, USA), as described previously by Chandrasekhar et al. (9). The optical density was then immediately measured at 530 and $590 \mathrm{~nm}$. The concentration of S-GAG was calculated using the standard curve for chondroitin sulfate from shark cartilage (Seikagaku, Tokyo) ranging from 0.3125 to $20 \mu \mathrm{M}$. When chondrocytes were treated with rhIL- $1 \alpha$ for $24 \mathrm{~h}$, the SGAG levels in the conditioned medium were significantly higher than those of the untreated control (Table 1). Rhein

Table 1. Effects of rhein and naproxen on the rhIL-1 $\alpha$-induced proteoglycan release

\begin{tabular}{lcc}
\hline Treatment & Conc. $(\mu \mathrm{M})$ & S-GAG $(\mu \mathrm{g} / \mathrm{ml})$ \\
\hline None & - & $9.58 \pm 1.17^{\# \#}$ \\
rhIL-1 $\alpha(1 \mathrm{ng} / \mathrm{ml})$ & - & $13.33 \pm 1.90$ \\
+ Rhein & 3 & $10.71 \pm 2.24$ \\
+ Rhein & 10 & $9.48 \pm 1.33^{*}$ \\
+ Rhein & 30 & $9.63 \pm 1.98^{*}$ \\
+ Naproxen & 1 & $13.78 \pm 1.72$ \\
\hline
\end{tabular}

Confluent chondrocytes were treated for $24 \mathrm{~h}$ with drugs in the presence of rhIL-1 $\alpha$, and then sulfated glycosaminoglycan (S-GAG) in the conditioned medium was detected by colorimetric assay using 1,9-dimethylmethylene blue. Each value represents the mean \pm S.D. for 5 independent experiments. $* P<0.05$, significantly different from rhIL-1 $\alpha$-treated control group by Dunnett's test. ${ }^{\# \#} P<0.01$, significantly different from the rhIL- $1 \alpha$-treated control group by Student's $t$-test.
(10 and $30 \mu \mathrm{M}$ ) significantly inhibited the rhIL- $1 \alpha$-induced S-GAG release $(28.9 \%$ inhibition at $10 \mu \mathrm{M}$ and $27.8 \%$ inhibition at $30 \mu \mathrm{M})$. Naproxen $(1 \mu \mathrm{M})$ had no effect. The stimulatory effect of IL-1 on the proteoglycan degradation and its inhibitory effect on the synthesis of the new proteoglycan were not inhibited by NSAIDs (10). It is well known that many proteinases, such as MMPs, cysteine proteinases and serine proteinases, and reactive oxygen species (ROS), such as hydroxyl radicals, hydrogen peroxide, superoxide and nitric oxide (NO), which are found in connective tissues and inflammatory cells, can degrade cartilage matrix components. Numerous studies have demonstrated that MMPs are most frequently implicated in the destruction of articular cartilage in arthritic diseases. Indeed, the level of MMPs, tissue inhibitors of MMPs (TIMPs), and proinflammatory cytokines in the serum and the synovial fluid of patients with OA or RA have been shown to significantly increase (7). Furthermore, proinflammatory cytokines induce and/or augment the production of proMMPs.

We determined the amount of proMMP-3 in the conditioned medium using the MMP-3 enzyme immunoassay (EIA) system (Amersham Pharmacia Biotech, Tokyo) according to the manufacturer's instructions. When chondrocytes were treated with rhIL-1 $\alpha$ for $24 \mathrm{~h}$, the production of proMMP-3 increased by about 34 -fold as compared with that of the untreated control (Table 2). Rhein (10 and $30 \mu \mathrm{M})$ significantly inhibited the production of proMMP$3(32.0 \%$ inhibition at $10 \mu \mathrm{M}$ and $56.7 \%$ inhibition at $30 \mu \mathrm{M})$. Naproxen had no effect.

The activities of MMPs are controlled at several levels including proenzyme activation and regulation by the specific MMPs inhibitors, TIMPs. We investigated whether the effect of rhein resulted in the decrease in MMPs activity. The apparent activity of the MMPs in the conditioned medium was determined using fluorescein isothiocyanate

Table 2. Effects of rhein and naproxen on the rhIL-1 $\alpha$-induced proMMP-3 production

\begin{tabular}{lcc}
\hline Treatment & Conc. $(\mu \mathrm{M})$ & proMMP-3 $(\mu \mathrm{g} / \mathrm{ml})$ \\
\hline None & - & $0.32 \pm 0.10^{\# \# \#}$ \\
rhIL-1 $\alpha(1 \mathrm{ng} / \mathrm{ml})$ & - & $10.89 \pm 1.08$ \\
+ Rhein & 3 & $9.53 \pm 2.32$ \\
+ Rhein & 10 & $7.41 \pm 1.36^{* *}$ \\
+ Rhein & 30 & $4.71 \pm 1.05^{* * *}$ \\
+ Naproxen & 1 & $11.59 \pm 3.12$ \\
\hline
\end{tabular}

Confluent chondrocytes were treated for $24 \mathrm{~h}$ with drugs in the presence of rhIL- $1 \alpha$, and then the proMMP-3 concentration in the conditioned medium was assayed using the EIA system. Each value represents the mean \pm S.D. for 5 independent experiments. ${ }^{* *} P<0.01$ and $* * * P<0.001$, significantly different from the rhIL- $1 \alpha$-treated control group by Dunnett's test. ${ }^{\# \#} P<0.001$, significantly different from the rhIL-1 $\alpha$-treated control group by Aspin-Welch's test. 
(FITC)-labeled casein as a substrate according to the method of Twining (11) with slight modifications of the microassay. Before the assay, proMMPs in the conditioned medium were activated by L-1- $p$-tosylamido-2-phenylethyl chloromethyl ketone treated-trypsin followed by the addition of a four-molar excess of soybean trypsin inhibitor. The enzyme activity that digests $1 \mu \mathrm{g}$ of casein for $1 \mathrm{~h}$ at $37^{\circ} \mathrm{C}$ was expressed as $1 \mathrm{U}$. Recombinant hIL- $1 \alpha$ significantly augmented the caseinolytic activity compared to the untreated control (Table 3). Rhein (10 and $30 \mu \mathrm{M})$ significantly suppressed the rhIL- $1 \alpha$-enhanced caseinolytic activity $(14.3 \%$ inhibition at $10 \mu \mathrm{M}$ and $37.7 \%$ inhibition at $30 \mu \mathrm{M})$. Naproxen had no effect. Next, the MMP-9 activity was determined using the MMP-9 activity assay system (Amersham Pharmacia Biotech) according to the manufacturer's instructions. The MMP-9 activity also significantly increased as compared with that of the untreated control (Table 3). Rhein (30 $\mu \mathrm{M})$ significantly inhibited the MMP9 activity (11.8\% inhibition). Naproxen had no effect.

The MMPs are well known to degrade proteoglycan. In this communication, we have shown that rhein suppresses the rhIL- $1 \alpha$-induced S-GAG release along with a decrease in production of proMMP-3. This is the first evidence that rhein down-regulates the proMMP-3 production in rabbit articular chondrocytes that was determined with an enzyme immunoassay system. The proMMPs suppression of rhein may be involved in the inhibition of S-GAG release from chondrocytes.

The mechanism by which rhein inhibits the proteoglycan degradation and proMMPs production is not fully understood at present. Cruz et al. (12) reported that the inhibition of the activator protein-1 activity might serve as the mechanism of diacerein that inhibits collagenase expression in bovine articular chondrocytes, but the action of rhein is not specified. On the other hand, it has been suggested that blocking the ROS production or decreasing the ROS levels reduces the IL-1-induced collagenase expression in the chondrocytes (13). Rhein is known to reduce the production of superoxide (14) and NO (15). Taken together, it is likely that rhein modulates the proMMPs production and activity via interference with multiple signaling pathways and thus protects the cartilage. Rhein may have a direct protective role on chondrocytes, or an indirect effect through inhibition of the proMMPs production. Further study is necessary to clarify the involvement of rhein induced proMMPs suppression in proteoglycan degradation.

To conclude, rhein, an active metabolite of diacerein, inhibits the destruction of articular cartilage induced by rhIL$1 \alpha$. These findings may be related to the suppressive effect of rhein on the proMMPs production.

\section{REFERENCES}

1 Marcolongo R, Fioravanti A, Adami S, Tozzi E, Mian M and Zampieri A: Efficacy and tolerability of diacerhein in the treatment of osteoarthrosis. Curr Ther Res 43, 878 - 887 (1988)

2 Nguyen M, Dougados M, Berdah L and Amor B: Diacerhein in the treatment of osteoarthritis of the hip. Arthritis Rheum 37, $529-536$ (1994)

3 Brandt KD, Smith G, Kang SY, Myers S, O'Connor B and Albrecht M: Effects of diacerhein in an accelerated canine model of osteoarthritis. Osteoarthritis Cartilage 5, 438 - 449 (1997)

4 Moore AR, Greenslade KJ, Alam CA and Willoughby DA: Effects of diacerhein on granuloma induced cartilage breakdown in the mouse. Osteoarthritis Cartilage 6, 19-23 (1998)

5 Debord P, Louchahi K, Tod M, Cournot A, Perret G and Petitjean O: Influence of renal function on the pharmacokinetics of diacerein after a single oral dose. Eur J Drug Metab Pharmacokinet 19, 13 - 19 (1994)

6 Nicolas P, Tod M, Padoin C and Petitjean O: Clinical pharmacokinetics of diacerein. Clin Pharmacokinet 35, 347 - 359 (1998)

7 Hembry RM, Bagga MR, Reynolds JJ and Hamblen DL: Immunolocalisation studies on six matrix metalloproteinases and their inhibitors, TIMP-1 and TIMP-2, in synovia from patients with osteo- and rheumatoid arthritis. Ann Rheum Dis 54, 25-32 (1995)

Table 3. Effects of rhein and naproxen on the rhIL-1 $\alpha$-enhanced caseinolytic and MMP-9 activity

\begin{tabular}{lccc}
\hline Treatment & Conc. $(\mu \mathrm{M})$ & Caseinolytic activity $(\mathrm{U} / \mathrm{ml})$ & MMP-9 activity $(\mathrm{U} / \mathrm{ml})$ \\
\hline None & - & $0.02 \pm 0.02^{\# \# \#}$ & $5.66 \pm 0.14^{\# \#}$ \\
rhIL-1 $\alpha(1 \mathrm{ng} / \mathrm{ml})$ & - & $1.54 \pm 0.06$ & $6.62 \pm 0.47$ \\
+ Rhein & 1 & $1.62 \pm 0.04$ & $\mathrm{NT}$ \\
+ Rhein & 3 & $1.46 \pm 0.12$ & $6.42 \pm 0.30$ \\
+ Rhein & 10 & $1.32 \pm 0.08^{*}$ & $6.18 \pm 0.36$ \\
+ Rhein & 30 & $0.96 \pm 0.06^{* *}$ & $5.84 \pm 0.50^{*}$ \\
+ Naproxen & 1 & $1.41 \pm 0.16$ & $7.05 \pm 1.35$ \\
\hline
\end{tabular}

Confluent chondrocytes were treated for $24 \mathrm{~h}$ with drugs in the presence of rhIL-1 $\alpha$, and then the caseinolytic or MMP-9 activity in the conditioned medium was determined using FITC-labeled casein or the MMP-9 activity assay system. Each value represents the mean \pm S.D. for five independent experiments. ${ }^{*} P<0.05$ and ${ }^{*} P<0.01$, significantly different from the rhIL- $1 \alpha$-treated control group by Dunnett's test. ${ }^{\# \#} P<0.01$ and ${ }^{\# \#} P<0.001$, significantly different from the rhIL- $1 \alpha$-treated control group by Aspin-Welch's test. NT: not tested. 
8 Dean DD: Proteinase-mediated cartilage degradation in osteoarthritis. Semin Arthritis Rheum 20, 2 - 11 (1991)

9 Chandrasekhar S, Esterman MA and Hoffman HA: Microdetermination of proteoglycans and glycosaminoglycans in the presence of guanidine hydrochloride. Anal Biochem 161, $103-108$ (1987)

10 Arner EC and Pratta MA: Independent effects of interleukin-1 on proteoglycan breakdown, proteoglycan synthesis, and prostaglandin $\mathrm{E}_{2}$ release from cartilage in organ culture. Arthritis Rheum 32, 288 - 297 (1989)

11 Twining SS: Fluorescein isothiocyanate-labeled casein assay for proteolytic enzymes. Anal Biochem 143, 30 - 34 (1984)

12 Cruz TF, Tang J, Pronost S and Pujol JP: Molecular mechanisms implicated in the inhibition of collagenase expression by diacer- heine. Rev Praticien 46, S15 - S19 (1996)

13 Lo YY, Conquer JA, Grinstein S and Cruz TF: Interleukin- $1 \beta$ induction of c-fos and collagenase expression in articular chondrocytes: involvement of reactive oxygen species. J Cell Biochem 69, 19-29 (1998)

14 Mian M, Brunelleschi S, Tarli S, Rubino A, Benetti D, Fantozzi $\mathrm{R}$ and Zilletti L: Rhein: an anthraquinone that modulates superoxide anion production from human neutrophils. J Pharm Pharmacol 39, 845 - 847 (1987)

15 Pelletier JP, Mineau F, Fernandes JC, Duval N and MartelPelletier J: Diacerhein and rhein reduce the interleukin $1 \beta$ stimulated inducible nitric oxide synthesis level and activity while stimulating cyclooxygenase-2 synthesis in human osteoarthritic chondrocytes. J Rheumatol 25, 2417 - 2424 (1998) 